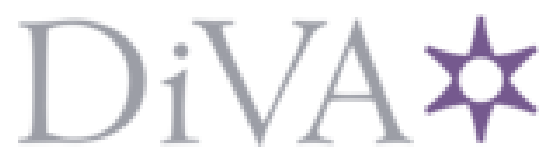

http://www.diva-portal.org

\title{
Postprint
}

This is the accepted version of a paper presented at IEEE PES General Meeting.

Citation for the original published paper:

Mahmood, F., Hooshyar, H., Vanfretti, L. (2017)

Sensitivity Analysis of a PMU-Fed Steady State Model Synthesis Method for Active Distribution Networks.

In: IEEE

N.B. When citing this work, cite the original published paper.

Permanent link to this version:

http://urn.kb.se/resolve?urn=urn:nbn:se:kth:diva-202324 


\section{Sensitivity Analysis of a PMU-Fed Steady State Model Synthesis Method for Active Distribution Networks}

\author{
Farhan Mahmood \\ KTH Royal Institute of Technology \\ Stockholm, Sweden \\ Email: farhanm@kth.se
}

\author{
Hossein Hooshyar \\ KTH Royal Institute of Technology \\ Stockholm, Sweden \\ Email: hosseinh @ kth.se
}

\author{
Luigi Vanfretti \\ KTH Royal Institute of Technology \\ Stockholm, Sweden \\ Email: luigiv@kth.se
}

\begin{abstract}
The purpose of this paper is to carry out sensitivity analysis of a method that performs steady state model synthesis (SSMS) of active distribution networks using Syncrophasor Measurements. An extended version of the Total Vector Error (TVE) is used as a metric to evaluate how sensitive the output of the SSMS method is to the changes in the inputs to the method. Location of PMUs in the system, system operating point, and occurrence of different disturbances are considered as the input to the SSMS method. The sensitivity analysis is performed through several case studies each containing changes in one of the inputs.
\end{abstract}

Index Terms-- active distribution network, model synthesis, PMU, sensitivity analysis.

\section{INTRODUCTION}

Sensitivity analysis is the study of how the uncertainty in the output of a mathematical model or system can be distributed to different sources of uncertainty in its inputs [1]. A related practice is uncertainty analysis, which has a greater focus on uncertainty quantification and propagation of uncertainty. Ideally, uncertainty and sensitivity analysis should be run in tandem.

Sensitivity analysis of a method can be performed by recalculating its outputs for a considerable number of times under different assumptions and then analyzing how their accuracy is impacted. This study is useful for a variety of purposes including

- Testing the robustness of the outputs in the presence of various uncertainties.

- Identifying the method's inputs that cause substantial uncertainty in the outputs and should therefore be considered for enhancing robustness.

- Searching for different types of errors in the outputs.

\section{A. Motivation and Previous Work}

With the increase of intermittent generation sources in distribution networks, it is becoming necessary for Transmission System Operators (TSOs) to have an accurate model of their distribution networks. Measurements from
Phasor Measurement Unit (PMU) from multiple locations in the distribution network can be exploited to be used for model synthesis of active distribution networks. In this context, a steady state model synthesis (SSMS) method is introduced in [2] which synthesizes a three-phase steady state equivalent model of the distribution network. Although a performance analysis of the method is presented in [2] using hardware-inthe-loop (HIL) simulation setup, yet there was a need to perform a detailed sensitivity analysis of the proposed SSMS method to know how sensitive the output of the method is to the changes in its inputs.

The application of sensitivity analysis in power system has been extensively discussed in literature [3] [4] [5]. In [3], sensitivity analysis is applied to evaluate the variation of total generation cost with respect to change in reactive power, for determining the optimal location of the capacitor banks in the network. The influence of parameter perturbations on power system stability limit has been discussed in [4]. In [5], a sensitivity analysis on the accuracy of different dc power-flow models is presented.

\section{B. Paper Contributions}

In this paper, a sensitivity analysis of the SSMS method (presented in [2]) has been carried out. Moreover, the paper proposes a methodology to perform sensitivity analysis on PMU data-based applications such as the proposed SSMS method.

The paper begins by summarizing the concept of steady state model synthesis in Section II. In Section III, a methodology to perform sensitivity analysis on the SSMS application is presented. Section IV describes the HIL realtime simulation setup used for sensitivity analysis of the SSMS application. Section V presents the results from the sensitivity analysis on the SSMS application. Conclusions are drawn in Section VI.

\section{The STEAdy StATE Model SyNTHESIS METHOD}

Assuming that PMU measurements are available at the boundary buses of a distribution network, a three-phase steady state equivalent model can be synthesized as illustrated in Fig. 1. As the operating conditions of the system changes, the 
parameters of the equivalent model are updated in real-time. The readers are referred to [2] for the details of the SSMS method.

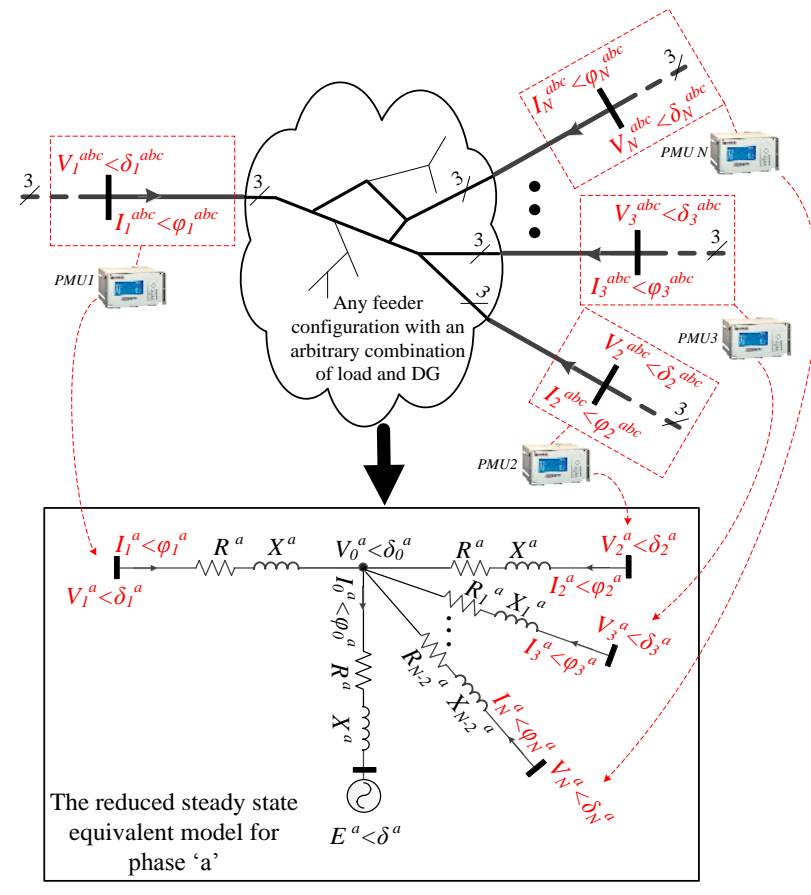

Fig. 1. Synthesized model based on ' $N$ ' number of PMU measurement points

\section{METHODOLOGY FOR SENSITIVITY ANALYSIS OF THE SSMS METHOD}

The main outputs of the SSMS method, summarized in the previous section, are the voltage and current phasors reproduced by the synthesized model. This section presents a methodology to analyze how the accuracy of these outputs is influenced by different inputs of the SSMS method.

\section{A. End-to-End Total Vector Error (TVE)}

The accuracy of the SSMS outputs can be measured using an extended revision of the TVE concept introduced in [6]. This extended revision, called end-to-end TVE in this study, is defined as the measure of the difference between the theoretical phasor value of the signal being measured and the reproduced version of the same phasor, as shown below

$$
T V E_{\text {end-to-end }}(n)=\sqrt{\frac{\left(\hat{V}_{r}(n)-V_{r}(n)\right)^{2}+\left(\hat{V}_{i}(n)-V_{i}(n)\right)^{2}}{V_{r}(n)^{2}+V_{i}(n)^{2}}}
$$

Where

$\hat{V}_{r}(n)=$ Real part of the reproduced voltage

$V_{r}(n)=$ Real part of the true (actual) voltage

$\hat{V}_{i}(n)=$ Imaginary part of the reproduced voltage

$V_{i}(n)=$ Imaginary part of the true (actual) voltage
As illustrated in Fig. 2, the end-to-end TVE consists of the following two TVEs:

\section{a. PMU TVE}

The difference between the "true" phasor, that is in our case existing in the simulation environment, and the phasor estimated by the PMU is defined as PMU TVE as shown in Fig. 2. This TVE can occur due to the phase angle error that is the product of instrumentation channels and improper timing sources of the PMU [7]. In our experimental setup, explained in Section IV, the real-time simulator and the PMUs have different dynamic range on voltage and current ratings. This can also contribute as a source of PMU TVE. In addition, the current amplifiers are sources of phase angle errors, which in turn may cause PMU TVE.

\section{b. Field Application TVE}

The difference between the phasor estimated by the PMU and the phasor reproduced by the synthesized model is defined as Field Application TVE. This type of TVE is mainly the product of the SSMS application estimation errors.

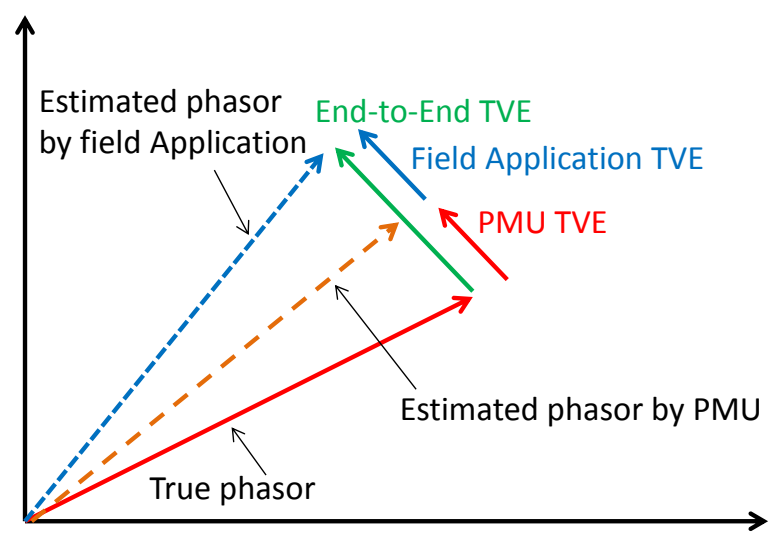

Fig. 2. Types of TVEs

\section{B. Methodology for Calculating End-to-End TVE}

The end-to-end TVE can be calculated by running the SSMS application in a HIL simulation setup (explained in Section IV). The HIL setup is used for real-time simulation of the grid model whose synthesized reduced model is of interest. As shown in Fig. 3, through the HIL setup, the estimated voltage and current phasors at the boundary buses of the grid model are fed to the SSMS application which estimates the parameters of the reduced equivalent model. These parameters are sequentially updated in real-time as soon as any change in the operating point of the system occurs. The resulting array of the parameters is fed to a reduced equivalent version of the grid model. The reduced model is then simulated to reproduce the corresponding voltage and current phasors. The reproduced voltage and current phasors are then compared to their true values in order to calculate the end-to-end TVE using (1). 
Note that as indicated in Fig. 3, the SSMS sensitivity analysis is performed by calculating the end-to-end TVE versus different inputs such as the location of PMUs, system operating points, and occurrence of different disturbances.

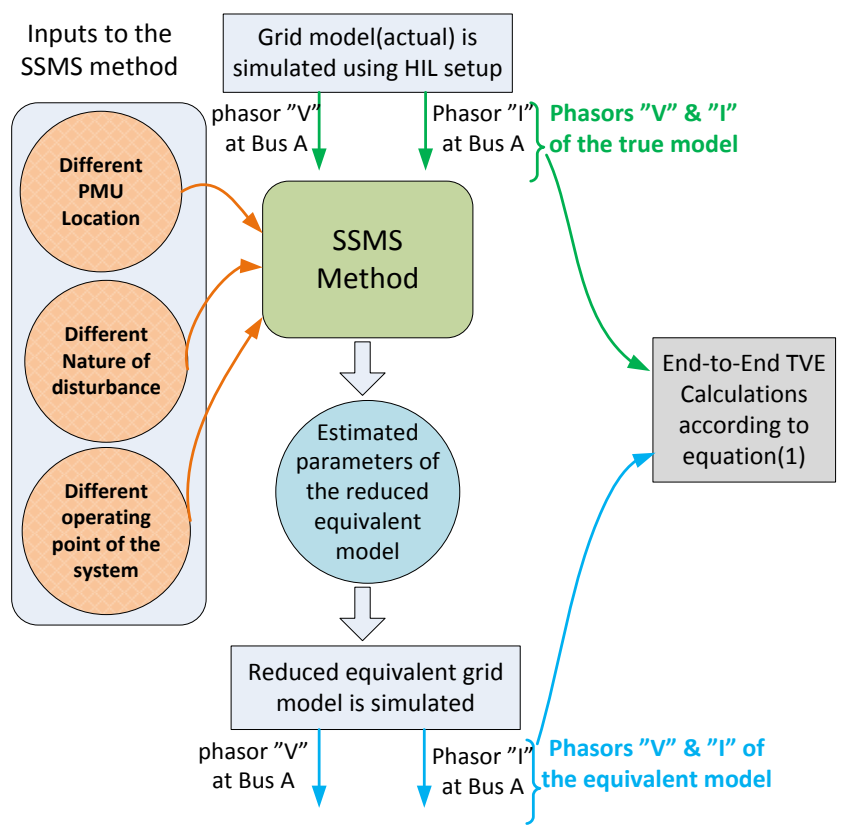

Fig. 3. Methodology for calculating end-to-end TVE

\section{End-to-End TVE as Evalution Metric}

As mentioned before, the end-to-end TVE is selected in this study as the evaluation metric for the sensitivity analysis of the results produced by the SSMS method.

We have defined a $3 \%$ mean error requirement for the endto-end $\mathrm{TVE}^{1}$ in this study. In other words, we would like the SSMS outputs, i.e. the reproduced phasors, to have limited sensitivity to the changes in the SSMS inputs (i.e. location of PMUs, system operating points, and occurrence of different disturbances) such that the end-to-end TVE remains within $3 \%$. The basis of this requirement is as follows:

- DC Power flow models available in literature can have more than $15.7 \%$ error [5].

- As SSMS is a method to synthesize steady state models of the active distribution network, having 3\% end-to-end TVE for estimated parameters still provides much better accuracy of the reduced equivalent models than power flow models.

- $3 \%$ is an acceptable value because end-to-end TVE contains PMU TVE, which is not related to the performance of the target application. Specifically, in our case, the amount of phase angle error generated by instrumentation and difference in dynamic range of the

\footnotetext{
${ }^{1}$ Observe that requirements in terms of each type of TVE defined could be imposed and assessed. However, such work would require additional experimental tests. Further work in this area will be carried out in future publications.
}

ratings of different equipment increase the end-to-end TVE.

\section{HIL REAL-TIME SimUlation SETUP}

This section describes the HIL real-time simulation setup for accessing the performance of SSMS method. As shown in Fig. 4, a grid model is simulated using the OPAL-RT real-time simulator. The measured voltages and currents are fed to PMUs through the analogue output ports of the OPAL-RT simulator and the amplifiers. As the figure shows, Syncrophasors are sent to a Phasor Data Concentrator (PDC) which streams the data over TCP/IP to a workstation computer holding Statnett's Synchrophasor Development Kit ( $\left.\mathrm{S}^{3} \mathrm{DK}\right)$ [8]. $S^{3} \mathrm{DK}$ serves as a real-time data mediator that parses the PDC data stream and makes it available to the SSMS application in LabVIEW environment. As shown in Fig. 4, different types of TVEs explained in Section III can be visualized.

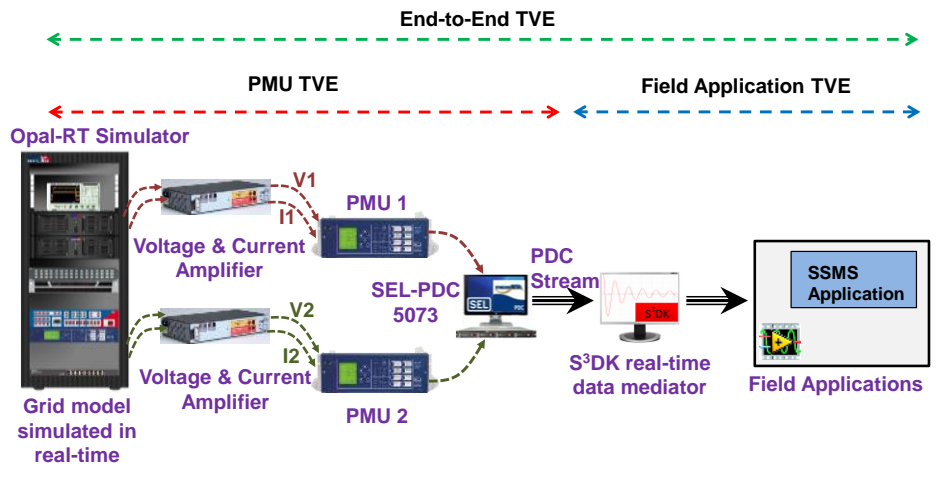

Fig. 4. HIL simulation setup showing different types of TVEs

\section{SEnsativity AnAlysis OF The SSMS Method}

Fig. 5 shows a single diagram of a grid network model, which has been adopted from an active distribution network model, presented in [9]. The grid model is used to carry out different case studies to show how sensitive the output of the SSMS method is with respect to the changes in its inputs. In [2], SSMS method is validated by the grid model, shown in Fig 5. Therefore, any changes in the grid model can be

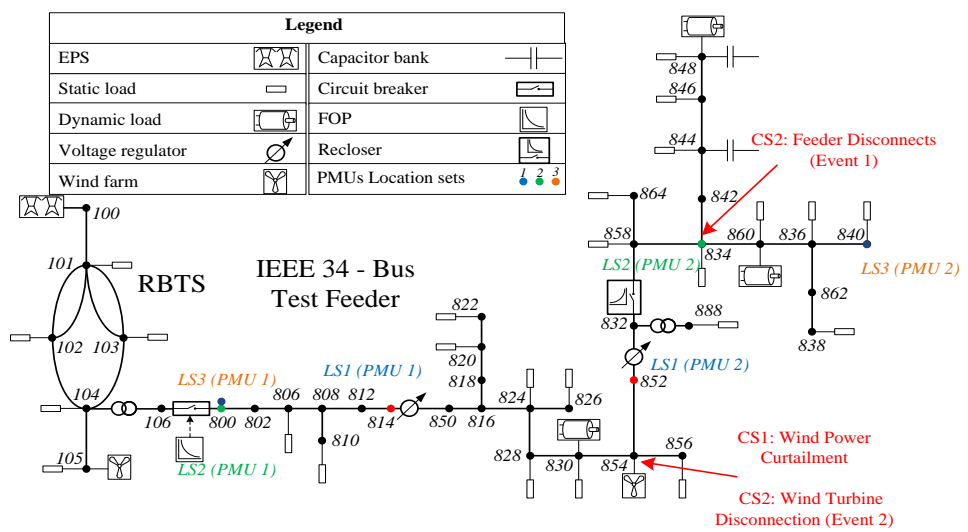

Fig. 5. Single-line diagram of a grid network 
considered as the inputs to the SSMS method. Three variable inputs are considered in this study: change in the system operating point, occurrence of different disturbances in the grid network, and change in the location of the PMUs installed in the network. For case study 1, the experiment is repeated 125 times (for each step decrease in wind power). Whereas, for case study 2 and case study 3, the experiment is repeated 40 times each. The sensitivity of the output of the SSMS method to these inputs is shown in the following case studies.

\section{A. Case Study 1: Change in system operating point}

This Case Study shows how sensitive the output of SSMS method is with respect to changes in the system operating point. The changes are realized by applying a power decrease of $0.35 \mathrm{MW}$ ( $16 \%$ of the total load) in 5 steps $(0.075 \mathrm{MW}$ in each step) to the wind turbine connected at node 854 in Fig. 5. The sensitivity of the output of the SSMS method is evaluated by calculating the mean, maximum and minimum values of the end-to-end TVE for each step decrease in the wind power generation. The results are summarized in Table I. The largest mean value of the end-to-end TVE for all the voltage and current phasors is $2.3723 \%$. Note that in this case study the PMUs are installed at nodes 814 and 852.

The standard deviation (SD) is calculated for each voltage and current phasor. The maximum value of SD of end-to-end TVE is $0.4644 \%$, which shows the uncertainty in the output of the SSMS method is small. The probability distributions for the end-to-end TVE for both voltage and current phasors, for both PMU 1 and PMU 2, are plotted and are shown in Fig. 6. The top plots correspond to the voltage phasors and the bottom plots are for the current phasors.

TABLE I

End-to-end TVE for Case Study 1

(Number of trails=125 for each step decrease in wind power)

\begin{tabular}{|c|c|c|c|c||}
\hline \multirow{2}{*}{ Phasors } & \multicolumn{4}{|c|}{ End-to-end TVE (\%) } \\
\cline { 2 - 5 } & Mean & Max & Min & SD \\
\hline \hline V1 & 0.1184 & 0.1564 & 0.1034 & 0.0143 \\
\hline \hline $\boldsymbol{I 1}$ & 2.3723 & 3.7165 & 1.6429 & 0.4644 \\
\hline \hline V2 & 1.6486 & 1.7288 & 1.5818 & 0.0416 \\
\hline \hline $\boldsymbol{I} 2$ & 1.4972 & 1.6172 & 1.4026 & 0.0559 \\
\hline \hline
\end{tabular}
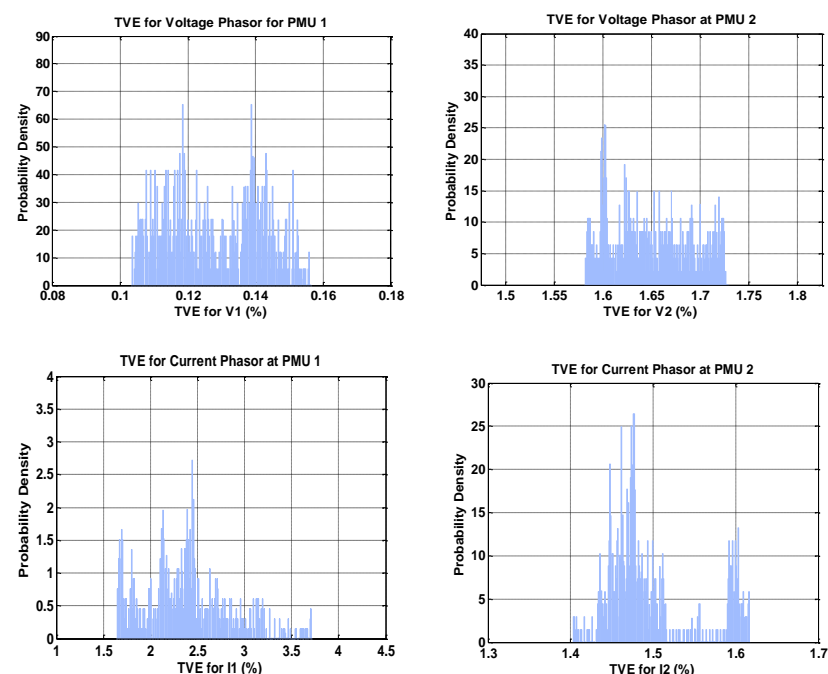

Fig. 6. Probability distribution of end-to-end TVEs for Case Study 1

\section{B. Case Study 2:Occurance of Disturbances}

The sensitivity of the output of the SSMS method is analyzed by applying two types of disturbances in the grid model (shown in Fig. 5). The following two events were created:

Event 1: Disconnection of a lateral feeder at bus 834

Event 2: Loss of $1 \mathrm{MW}$ wind generation at bus 854

The end-to-end TVE is calculated for three different time periods, listed in Table II, to analyze the sensitivity of the SSMS output with respect to these two events.

TABLE II

Different time periods in Case Study 2

\begin{tabular}{|c|l|}
\hline No. & Details of the Sections \\
\hline 1 & Before Event 1 \\
\hline 2 & Between Event 1 \& Event 2 \\
\hline 3 & After Event 2 \\
\hline
\end{tabular}

For each time period, mean, maximum and minimum values of the end-to-end TVE for each voltage and current phasor were calculated. Note that in this case study the PMUs are installed at nodes 814 and 852 . The results are summarized in Table III. As shown in the table, the largest mean value of the end-to-end TVE is $3.0549 \%$ for all the voltage and currents phasors for both PMUs. Moreover, SD is calculated for each phasor for both PMUs. The maximum value for SD of the end-to-end TVE, for all phasors of both PMUs is $0.4349 \%$.

The results from Table III show that the end-to-end TVE remains in the permissible range despite the occurrence of the two disturbances.

TABLE III

End-to-end TVE for Case Study 2

(Number of trails $=40$ )

\begin{tabular}{|c|c|c|c|c|c||}
\hline \multirow{2}{*}{ Phasors } & \multirow{2}{*}{ Sections } & \multicolumn{4}{|c|}{ End-to-end TVE (\%) } \\
\cline { 2 - 6 } & & Mean & Max & Min & SD \\
\hline \hline \multirow{3}{*}{$\boldsymbol{1}$} & 1 & 0.1267 & 0.1633 & 0.1 & 0.0251 \\
\cline { 2 - 6 } & 2 & 0.1382 & 0.1672 & 0.1051 & 0.0218 \\
\cline { 2 - 6 } & 3 & 0.1186 & 0.1486 & 0.0896 & 0.0193 \\
\hline \hline \multirow{3}{*}{$\boldsymbol{1}$} & 1 & 2.5192 & 2.9901 & 2.1016 & 0.2804 \\
\cline { 2 - 6 } & 2 & 3.0549 & 3.6330 & 2.3176 & 0.4349 \\
\cline { 2 - 6 } & 3 & 1.3455 & 1.6704 & 1.0952 & 0.1632 \\
\hline \hline \multirow{3}{*}{$\boldsymbol{2}$} & 1 & 1.6448 & 1.6830 & 1.611 & 0.0239 \\
\cline { 2 - 6 } & 2 & 1.6259 & 1.6650 & 1.6004 & 0.0211 \\
\cline { 2 - 6 } & 3 & 1.7326 & 1.7592 & 1.7024 & 0.0163 \\
\hline \hline \multirow{3}{*}{} & 1 & 1.4878 & 1.5671 & 1.4390 & 0.0265 \\
\cline { 2 - 6 } & 2 & 1.7434 & 1.7843 & 1.7063 & 0.0237 \\
\cline { 2 - 6 } & 3 & 1.5624 & 1.5862 & 1.5442 & 0.0120 \\
\hline \hline
\end{tabular}

\section{Case Study 3: Change in PMU Location}

In this case study, the location of each pair of PMUs (used in the SSMS method) is varied, as shown in the grid model in 
Fig 5. This is to investigate how sensitive the SSMS output is with respect to the measurement points, that is the PMU location. In this case study, the following Location Sets (LS) are selected:

- $\quad$ Location Set 1: PMU 1 at node 814 PMU 2 at node 852

- $\quad$ Location Set 2: PMU 1 at node 800 PMU 2 at node 834

- Location Set 3: PMU 1 at node 800 PMU 2 at node 840

For each PMU location set (LS), mean, maximum and minimum values of end-to-end TVE for voltage and current phasors for both PMU 1 and PMU 2 were calculated. The results are summarized in Table IV. As shown in the table, the largest mean value of the End-to-End TVE is $2.5192 \%$ for all the voltage and currents phasors for both PMUs. Moreover, SD is calculated for each LS for the voltage and the current phasors for both PMUs. The maximum value for SD for all the phasors for both PMUs is $0.2804 \%$.

The results from Table IV show that the end-to-end TVE does not change much by changing the PMU location set in the network, which indicates that the output of SSMS method is impervious to different PMU locations in the network.

TABLE IV

End-to-end TVE for Case Study 3

(Number of trails $=40$ )

\begin{tabular}{|c|c|c|c|c|c|}
\hline \multirow{2}{*}{ Phasors } & \multirow{2}{*}{$\begin{array}{c}P M U \\
\text { Location } \\
\text { Set }\end{array}$} & \multicolumn{4}{|c|}{ End-to-end TVE (\%) } \\
\hline & & Mean & $\operatorname{Max}$ & Min & $S D$ \\
\hline \multirow{3}{*}{$V 1$} & 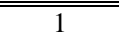 & 0.1267 & 0.1633 & 0.1 & 0.0251 \\
\hline & 2 & 0.0533 & 0.0609 & 0.0407 & 0.0056 \\
\hline & 3 & 0.0355 & 0.0383 & 0.03412 & 0.0013 \\
\hline \multirow{3}{*}{ II } & 1 & 2.5192 & 2.9901 & 2.1016 & 0.2804 \\
\hline & 2 & 2.4842 & 2.8366 & 1.8817 & 0.2727 \\
\hline & 3 & 1.6148 & 1.74208 & 1.54582 & 0.0630 \\
\hline \multirow{3}{*}{$V 2$} & $\overline{11}$ & 1.6448 & 1.6830 & 1.611 & 0.0239 \\
\hline & 2 & 1.6164 & 1.6623 & 1.5837 & 0.0243 \\
\hline & 3 & 1.5254 & 1.67248 & 1.64415 & 0.00833 \\
\hline \multirow{3}{*}{$I 2$} & 1 & 1.4878 & 1.5671 & 1.4390 & 0.0265 \\
\hline & 2 & 1.4667 & 1.4825 & 1.4476 & 0.01218 \\
\hline & 3 & - & - & - & - \\
\hline
\end{tabular}

\section{CONCLUSIONS}

This paper has presented a sensitivity analysis of a method that performs steady state model synthesis (SSMS) of active distribution networks using synchronized phasor measurement data. The original TVE concept was extended (end-to-end TVE) to serve as an evaluation metric in the sensitivity analysis. A methodology for sensitivity analysis of the SSMS application was presented and performed through three different case studies. The presented results show that the method's output adjusted to the changes in operating point of the system well and provided acceptable values for the end-to-end TVE before and after the disturbances. In addition, it was shown that the end-to-end TVE does not change much by changing the location of the PMUs in the grid. In summary, the results of the case studies show that the output of the SSMS method is quite impervious to the changes in its inputs, which indicates the robustness of the method.

\section{ACKNOWLEDGMENT}

This work was supported in part by the FP7 IDE4L project funded by the European Commission and the STandUp for Energy Collaboration Initiative.

\section{REFERENCES}

[1] Saltelli, A.; Ratto, M.; Andres, T.; Campolongo, F.; Cariboni, J.; Gatelli, D.; Saisana, M.; Tarantola, S. (2008). Global Sensitivity Analysis: The Primer. John Wiley \& Sons.

[2] F. Mahmood; H. Hooshyar; J. Lavenius; P. Lund; L. Vanfretti, "Realtime Reduced Steady State Model Synthesis of Active Distribution Networks Using PMU Measurements," in IEEE Transactions on Power Delivery, in press, early access available at http://ieeexplore.ieee.org/Xplore/home.jsp.

[3] J. Chureemart and P. Churueang, "Sensitivity analysis and its applications in power system improvements," Electrical Engineering/Electronics, Computer, Telecommunications and Information Technology, 2008. ECTI-CON 2008. 5th International Conference on, Krabi, 2008, pp. 945-948.

[4] Michele Brucoli, Bruno Maione, Ernesto Margarita and Francesco Torelli, "Sensitivity analysis in power system dynamic stability studies," Electric Power Systems Research, 1981. Elsevier Sequoia S.A., Lausanne, pp. 59-66.

[5] Yingying Qi; Di Shi; Tylavsky, D., "Impact of assumptions on DC power flow model accuracy" North American Power Symposium (NAPS), 2012, vol., no., pp.1-6, 9-11 Sept. 2012.

[6] IEEE Std C37.118, "IEEE standard for syncrophasor measurements for power systems" no. 2, pp. 1-2011, 2006.

[7] R. S. Singh, H. Hooshyar and L. Vanfretti, "Assessment of time synchronization requirements for Phasor Measurement Units," PowerTech, 2015 IEEE Eindhoven, Eindhoven, 2015, pp. 1-6.

[8] L. Vanfretti, V. H. Aarstrand, M. S. Almas, V. S. Peric, J. O. Gjerde, "A software development toolkit for real-time synchrophasor applications," in Proc. IEEE PowerTech, 2013.

[9] H. Hooshyar, F. Mahmood, L. Vanfretti, M. Baudette, "Specification, implementation, and hardware-in-the-loop real-time simulation of an active distribution grid," Elsevier Sustainable Energy, Grids and Networks (SEGAN), Volume 3, September 2015, Pages 36-51. 